position. A chapter devoted to optically active long chain compounds brings together the techniques applicable to the study of such compounds and the information obtained. Cyclopropane and cyclopropene fatty acids are similarly reviewed in a neat and readable chapter. Milk lipids are reviewed in depth, and the large volume of analytical data accumulating in this commercially important topic makes for heavy reading. Nevertheless, even perusal of a catalogue of the milk fats of seventy-five different species can be rewarding for the general reader, if only to challenge his imagination on how to milk a black rhinoceros.

In conclusion, this volume is an excellent reference source for the specialist, and as such is to be recommended. P. B. Garland

\section{ION-MOLECULE REACTIONS}

\section{Ion Molecule Reactions}

By E. W. McDaniel, V. Cermak, A. Dalgarno, E. E. Ferguson and L. Friedman. (Wiley Interscience Series in Atomic and Molecular Collisional Processes.) Pp. xiii + 374. (Wiley (Interscience): New York and London, August 1970.) 190s.

THE subjoct which this book treats has in the past few years been expanding so rapidly that a comprehensive review by notable specialists in the field is most welcome. If a verdict may be placed before the evidence, the book may at once be said to be a contribution to the literature which will be an important reference for many yoars, although other volumes on the same subject should be produced. Perhaps the only serious criticism which can be offered is that, after starting the project in 1964, some portions at least were probably completed by 1968, and a considerable and regrettable delay in its general availability seems to have occurred.

After a short introductory chapter, there follows in the second chapter a comprehensive review of the experimental method relevant to the study of ion-molecule reactions. The use and accurate control of electron bombardment and other similar ion sources is first described, followed by accounts of their adaptation to the measurement of rate coefficients of suitable reactions. Next follows a section on the sampling of ions from electrical discharges and afterglows. These systems, which in some cases mimic the conditions of the upper atmosphere, must be vory spccially controlled in order to produce reliable values of rate coefficients. These problems of control and some of the solutions adopted are fully described. The section on the "flowing afterglow" technique is of notable length and completeness.

The chapter continues with a section on drift tube techniques which includes a full treatment of the problem of diffusion in conditions of low $\mathrm{E} / \mathrm{P}$ with simultaneous reaction of the ions. There is then a section describing what would now be called the earlier work of the relatively new field of ion cyclotron resonance. The chapter concludes with a lengthy and valuable review of the information obtained using beam techniques. This includes the description of classical kinematics which is needed for a proper understanding of the results.

The experimental tone of chapter two is continued in chapter four, which gives a review of existing data under the heading "Reaction Rates from Ionospheric and Airglow Data". This chapter, however, quotes only two references dated later than 1967 , and those readers wishing for fully up to date information will have to search further.

The fifth chapter is headed "Ion-molecule Chemistry" and seeks to bring these reactions more fully into the gaze of chemists by discussing them in terms of heats of formation of the ions, orders of reaction and the dependence of rate coefficient on energy. A discussion of dependence of rate on system temperature is notably absent from the subject.

The compilation of "Quantitative Data" in chapter six lists all known data on cross-sections and/or rate coefficient, and for each reaction supplies also the "Langevin" rate coefficient for comparison. Tables are given for four classes of reaction. There is then a section in which graphical data on various reactions are assembled. This chapter shows several references dated 1969, plus several results "unpublished" or "to be published", and provides a very valuable collection.

The third chapter is reserved for special mention, because this treatment of the theory of ion-molecule collisions is probably the material least expected by a potential reader already well versed in the subject. Running to nearly a hundred pages, or a quarter of the book, it gives a treatment based on quantum theory which is full without being overwhelming, and is very well timed in its appearance. Improvements in experimental technique have, over several years, made tho classical Langevin treatment seem inadequate. The patchwork which has thus developed may, with such a stimulus as the introduction of this chapter provides, ovolve into a more coherent and generally satisfactory discussion of the phenomena.

P. F. KNEWSTUBB

\section{FAMOUS INEQUALITIES}

Finite Sections of Some Classical Inequalities

By Herbert S. Wilf. (Ergebnisse der Mathematik und ihrer Grenzgebiete, Band 52.) Pp. vii +83 . (SpringerVerlag: Berlin and New York, 1970.) 28 DM; \$7.70.

THE problem here investigated may be elucidated by a simple example. For positive $a_{n}$, it is known that

$$
\sum_{1}^{\infty}\left\{\left(a_{1}+a_{2}+\ldots+a_{n}\right) / n\right\}^{2} \leq 4 \sum_{1}^{\infty} a_{n}^{2}
$$

where the constant 4 is best possible, that is, cannot be replaced by a smaller number. If now the sums are taken not to $\infty$ but to an integer $N$, say, then we may hope to replace 4 by a constant $k(N)$, where $k(N) \rightarrow 4$ as $N \rightarrow \infty$. The examination of the character of $k(N)$ for a number of the classical inequalities brings together scveral analytical techniques: asymptotic methods, Toeplitz forms and integral kernels, functional analysis with operators which are not necessarily linear. Hilbert's famous inequality

$$
\sum_{m, n}^{\infty}\left\{a_{m} a_{n} /(m+n)\right\}<\pi \sum^{\infty} a_{m}^{2}
$$

belongs essentially to linear operator theory, and a great deal is known about its corresponding $k(N)$; for instance,

$$
k(N)=\pi-\pi^{8} / 2(\log N)^{2}+\mathrm{O}\left\{(\log \log N) /(\log N)^{3}\right\}
$$

On the other hand, non-linear theory is involved in Carlemann's inequality

$$
\stackrel{\infty}{\Sigma}^{\infty}\left(a_{1} a_{2} \ldots a_{n}\right)^{1 / n}<e \sum^{\infty} a_{n}
$$

and here the first discussion of the appropriate $k(N)$ is to be found in a delicate and ingenious argument given by de Bruijn in 1963.

The author has provided a concise and lucid account of the present state of knowledge, with a useful biblio. graphy. The young postgraduate worker interested in "hard" analysis will find here many suggestions for further research.
T. A. A. BroAdBent 